J Psychosom Res. 2009 December ; 67(6): 497-501. doi:10.1016/j.jpsychores.2009.09.002.

\title{
Clinical course of Tourette syndrome ${ }^{\star}$
}

\author{
Michael H. Bloch* and James F. Leckman \\ Yale Child Study Center, Yale University School of Medicine, New Haven, CT, USA
}

\begin{abstract}
Objective-Tourette syndrome (TS) is a childhood-onset neuropsychiatric disorder characterized by multiple motor and vocal tics lasting at least a year in duration. Children with TS often experience comorbid conditions such as obsessive-compulsive disorder (OCD) and attentiondeficit disorder. The goal of this article was to review the long-term clinical course of tics and comorbid conditions in children with TS.
\end{abstract}

Method-We conducted a traditional literature search to locate relevant articles regarding longterm outcome and prognosis in TS and tic disorders.

Results-Tics typically have an onset between the ages of 4 and 6 years and reach their worstever severity between the ages of 10 and 12 years. On average, tic severity declines during adolescence. By early adulthood, roughly three-quarters of children with TS will have greatly diminished tic symptoms and over one-third will be tic free. Comorbid conditions, such as OCD and other anxiety and depressive disorders, are more common during the adolescence and early adulthood of individuals with TS than in the general population.

Conclusion-Although tics are the sine qua non of TS, they are often not the most enduring or impairing symptoms in children with TS. Measures used to enhance self-esteem, such as encouraging strong friendships and the exploration of interests, are crucial to ensuring positive adulthood outcome in TS.

\section{Keywords}

Tourette syndrome; Clinical course; Prognosis

\section{Introduction}

Tourette syndrome (TS) is a childhood-onset neuropsychiatric disorder characterized by multiple motor and vocal tics that last at least a year in duration [1]. Once thought to be a rare condition, TS is known to affect four to six in every 1000 children [2-5]. Transient tic disorder, characterized by tics that are present for less than a year in duration, and chronic tic disorders, which are characterized by either motor or vocal tics, but not both, have a lifetime prevalence of approximately $20 \%$ and 5\%, respectively, in children [2-5]. Individuals with TS commonly experience comorbid psychiatric disorders such as attentiondeficit hyperactivity disorder (ADHD) and obsessive-compulsive disorder (OCD) [6]. These comorbid conditions often cause more impairment in individuals with TS than the tics themselves [7].

\footnotetext{
$\star$ The authors have no conflicts of interest to disclose.

(C) 2009 Published by Elsevier Inc.

*Corresponding author. Child Study Center, Yale University School of Medicine, PO Box 2070900, New Haven, CT 06520, USA. michael.bloch@yale.edu (M.H. Bloch).
} 
The purpose of this article was to discuss the clinical course of individuals with TS. The article will begin by focusing on the course of tic symptoms in TS, then focus on the clinical course of common comorbid conditions and finish by discussing adulthood global functioning and psychosocial outcome of children with TS. The conclusion will summarize, for clinicians, the prognostic information we use to explain the expected clinical course of TS to families seeking evaluation in our clinic.

\section{Tic symptoms}

A tic is a sudden, repetitive, nonrhythmic, stereotyped motor movement or vocalization involving discrete muscle groups [8]. Tics in TS typically begin around the age of 4-6 as simple motor movements such as eye blinking, nose twitching, or facial grimaces [9]. Motor tics usually progress in a rostrocaudal direction with time [9]. Vocal tics, when they appear, typically first manifest themselves a year or two after the onset of motor tics [9]. Vocal tics begin as simple vocalizations such as throat clearing, sniffing, or fractions of words [9]. With increasing age, both motor and vocal tics become more complex. Motor tics often evolve into more elaborate movements, and vocal tics often develop into words or phrases. Many TS patients experience premonitory urges [10]. A premonitory urge is a sensory phenomenon that occurs immediately prior to a tic, similar to the need to sneeze or itch [10]. Awareness of premonitory urges increases with age and is present in as many as $90 \%$ of adolescents with TS [11]. Tic symptoms of TS generally occur in bouts and wax and wane in severity over time [12]. While factors such as stress, anxiety, and fatigue are known to exacerbate tics in many individuals, focused concentration, especially involving fine-motor movements such as playing a musical instrument, dancing, or playing sports, alleviates tics [13]. Many TS patients are able to temporarily suppress tics, but often at the expense of concentration and exhaustion [14]. As children get older they typically develop a greater ability to suppress tics [14].

Fig. 1 demonstrates the clinical course of tic severity in a large cohort of 42 children with TS followed for an average of 7.3 years after initial evaluation [9].

When George Gilles de la Tourette originally described the clinical syndrome that now bears his name, tics were hypothesized to be persistent and lifelong; our current knowledge suggests this is far from the case [15]. Tics typically emerge around the age of 4-6 years, reach their worst-ever early in the second decade of life, and then, on average, improve during adolescence [16]. Fig. 2 describes the adulthood tic outcome in a combined cohort of 82 children followed from initial evaluation (average age 11 years) to follow-up in young adulthood [16,17]. As Fig. 2 demonstrates, over one-third of children with TS were completely tic free at follow-up, slightly less than half had minimal to mild tics, and less than a quarter had moderate or greater tics at follow-up. These results contrast to their worstever period where all individuals experienced at least moderate tics. Less than $5 \%$ of individuals reported experiencing worse tics in young adulthood than they did in childhood. Similar results of tic improvement have been demonstrated in a large cohort of children with tics and comorbid ADHD [18].

Clinical assessment data, neuropsychological testing, and structural neuroimaging have been examined to determine whether there are childhood correlates of tic severity outcomes in adulthood. Childhood clinical characteristics are rather poor determinants of future adulthood tic severity, with only current tic severity in early adolescence being weakly associated with adulthood tic severity [17]. Worst-ever tic severity and the presence of comorbid illnesses in childhood were not associated with tic severity in adulthood [17]. Poor fine-motor skills in childhood, as measured by the Purdue Pegboard test, were associated with increased adulthood tic severity [19]. Smaller caudate volume on childhood MRI scans was also associated with increased adulthood tic severity [20]. Childhood structural 
neuroimaging and neuropsychological testing data, although not robust enough to be a useful clinical prognosticator of outcome, does suggest particular neuropathological processes associated with persistent TS [21]. Specifically, abnormalities within corticostriatal-thalmico cortical circuits, possibly involving inhibitory interneurons within the basal ganglia, may be associated with the pathogenesis and persistence of many TS cases [22]. A full review of the neuropathology of TS is beyond the scope of this review but has been described in depth elsewhere [23-25].

The typical improvement of tic symptoms during adolescence has significant implications for the use of behavioral, pharmacological, and neurosurgical treatments in children with TS. The use of effective pharmacological treatments for TS (i.e., alpha- 2 agonists and antipsychotics) is employed to help relieve impairing tic symptoms. There is no evidence that suggests that these pharmacological treatments alter the long-term course of TS. Given the significant side effects of pharmacological agents, pharmacological treatment should be utilized to minimize the impact of tics on social and academic functioning, not necessarily to eliminate tics completely. Behavioral interventions such as habit-reversal therapy should be offered to all patients with impairing tics. Furthermore, more invasive interventions for TS such as deep brain stimulation and neurosurgery are strongly discouraged until well into adulthood, even for patients with impairing tics. This recommendation is made because many children's tics will improve with time regardless of treatment. A full review of the available treatments for TS is beyond the scope of this review but has been described well elsewhere [26,27].

\section{Comorbid conditions}

\section{Attention-deficit hyperactivity disorder}

In epidemiologic samples, greater than half of individuals with TS experience comorbid ADHD [6]. The prevalence of ADHD in patients with TS who reach clinical attention may be even higher [28]. When ADHD is present in individuals with TS, the ADHD symptoms typically precede the onset of tic symptoms and are associated with greater social, behavioral, and academic problems than the tics themselves [29,30]. Not surprisingly, comorbid ADHD symptoms in childhood have been associated with a decreased quality of life and global functioning in early adulthood for individuals with TS [31]. Unfortunately, studies examining the clinical course of ADHD, specifically in individuals with comorbid tics, are lacking. In the general ADHD population, however, it has been shown that the hyperactivity symptoms of ADHD generally improve during adolescence, whereas the inattention symptoms of ADHD often persist into adulthood [32].

Comorbid ADHD in children with TS often has a negative impact on concurrent social, academic, and behavioral function [29]. Comorbid ADHD symptoms can also negatively impact future quality of life and global psychosocial functioning [7]. Pharmacological treatment of impairing comorbid ADHD in children with TS is recommended [26]. A study conducted by the Tourette Syndrome Study Group demonstrated that both clonidine, an alpha-2 agonist, and methylphenidate, a psychostimulant, are effective treatments for ADHD in individuals with comorbid tics [33] The alpha-2 agonist, clonidine, appeared more effective in targeting tic symptoms than methylphenidate, and the combination of methylphenidate and clonidine was more effective than either treatment alone in treating both ADHD and tic symptoms. Case reports and clinical observations have suggested that methylphenidate may exacerbate tic symptoms in some individuals with TS [34]. For this reason, the pharmaceutical companies currently list tic symptoms as a contraindication to the use of most psychostimulant medications. However, all evidence from randomized clinical trials in children with comorbid tics suggests that these medications are, at worst, tic neutral on a population level [35]. 


\section{Obsessive-compulsive disorder}

Roughly one-third to one-half of children with TS will experience comorbid OCD throughout their lifetime [17]. OCD symptoms in patients with TS have an onset around the time that the tics reach their worst-ever, but symptoms may also appear de novo in adulthood. OCD patients with comorbid tics tend to have greater rates of symmetry obsessions, and counting, repeating, ordering, and arranging compulsions than OCD patients without comorbid tic symptoms [36]. In terms of pharmacotherapy, children with OCD and comorbid tics are likely to have a worse response to selective-serotonin reuptake inhibitors (SSRIs) than children without comorbid tics [37]. While children with OCD and comorbid tics may not respond optimally to SSRIs, it has been shown that children with OCD, with and without comorbid tics, appear to have a similar response to cognitive behavioral therapy [37]. Another treatment option for OCD patients with comorbid tics is antipsychotic medication. A meta-analysis of antipsychotic augmentation trials for treatment refractory OCD suggests that OCD patients with comorbid tics may have an improved response to this intervention [38].

Long-term outcome studies of children with TS suggest that increased IQ and smaller caudate nucleus volumes on childhood structural MRI scans may be associated with increased OC symptoms in early adulthood $[17,20]$. Adulthood outcome studies suggest that childhood OCD patients with comorbid tics may have an increased likelihood of remission of OCD symptoms by adulthood [39]. These data suggest that OC symptoms in children with OCD and comorbid tics may follow a similar developmental trajectory to the tics themselves. Fig. 3 depicts the typical clinical course of ADHD, OCD, and tic symptoms in children with TS [40].

\section{Other comorbid conditions}

Children with TS have higher rates of comorbid developmental disorders, learning disabilities, and disruptive behavior disorders than the general population [41]. The symptoms of these comorbid conditions typically appear prior to the time that tics reach clinical attention. When present, aggressive treatment of these conditions is warranted. Currently, there is no evidence to suggest that the recommended treatment of these conditions should differ between individuals with and without comorbid tic disorders. Explosive rage attacks can be particularly challenging in individuals with TS, but therapies such as parent management training and anger management training, which are effective in the general population, are also effective in TS $[42,43]$.

Children with TS experience comorbid depressive and anxiety disorders during the course of adolescence and early adulthood more frequently than unaffected controls [17,31]. Roughly $40 \%$ of children with TS will experience depression or a non-OCD anxiety disorder [31]. Children with TS and their parents should be educated to recognize the signs and symptoms of these conditions.

\section{Conclusions}

Table 1 reveals some psychoeducational clinical pearls that we use in evaluating children with TS. Children with TS typically experience a significant reduction in tic symptoms during adolescence. More than three-quarters of children with significant childhood tic symptoms will experience fewer and/or less severe tics in early adulthood and over one-third will be completely tic free in adulthood. In terms of comorbidity, a greater proportion of children with TS experience developmental disorders, ADHD, learning disorders, and disruptive behavioral disorders than among the general population. Symptoms of these conditions generally precede the onset of tics. Also, during adolescence and early adulthood 
children with TS are at greater risk of developing OCD and depressive disorders. Children with chronic or transient tic disorders appear to have a more benign comorbidity profile than children with TS [6]. In our clinical experience, developing strong interests and good friendships (sources of self-esteem) is helpful in ensuring good adulthood outcomes in children, especially those with tics.

\section{Acknowledgments}

We thank Kaitlyn E. Panza, B.A., for her help in revising this manuscript. The authors acknowledge the National Institute of Mental Health support of the Yale Child Study Center Research Training Program (MHB, JFL), K05MH076273 (JFL); the National Institute of Health Loan Repayment Program (MHB); the support of the Tourette's Syndrome Association, Inc. (JFL); and the APIRE/Eli Lilly Psychiatric Research Fellowship (MHB).

\section{References}

1. Diagnostic and statisticalmanual of mental disorders: DSM-IV-TR. Washington D.C: American Psychiatric Association; 2000.

2. Scahill L, Sukhodolsky DG, Williams SK, Leckman JF. Public health significance of tic disorders in children and adolescents. Adv Neurol. 2005; 96:240-8. [PubMed: 16383223]

3. Robertson MM. The prevalence and epidemiology of Gilles de la Tourette syndrome: Part 1 . The epidemiological and prevalence studies. J Psychosom Res. 2008; 65:461-72. [PubMed: 18940377]

4. Robertson MM. The prevalence and epidemiology of Gilles de la Tourette syndrome: Part 2. Tentative explanations for differing prevalence figures in GTS, including the possible effects of psychopathology, aetiology, cultural differences, and differing phenotypes. J Psychosom Res. 2008; 65:473-86. [PubMed: 18940378]

5. Khalifa N, von Knorring AL. Prevalence of tic disorders and Tourette syndrome in a Swedish school population. Dev Med Child Neurol. 2003; 45:315-9. [PubMed: 12729145]

6. Khalifa N, von Knorring AL. Psychopathology in a Swedish population of school children with tic disorders. J Am Acad Child Adolesc Psychiatry. 2006; 45:1346-53. [PubMed: 17075357]

7. Bernard BA, Stebbins GT, Siegel S, Schultz TM, Hays C, Morrissey MJ, et al. Determinants of quality of life in children with Gilles de la Tourette syndrome. Mov Disord. 2009; 24:1070-3. [PubMed: 19306279]

8. American Psychiatric Association. Diagnostic and Statistical Manual of Mental Disorders. Washington, DC: American Psychiatric Association Press; 2000.

9. Leckman JF, Zhang H, Vitale A, Lahnin F, Lynch K, Bondi C, et al. Course of tic severity in Tourette syndrome: the first two decades. Pediatrics. 1998; 102(1 Pt 1):14-9. [PubMed: 9651407]

10. Leckman JF, Walker DE, Cohen DJ. Premonitory urges in Tourette's syndrome. Am J Psychiatry. 1993; 150:98-102. [PubMed: 8417589]

11. Woods DW, Piacentini J, Himle MB, Chang S. Premonitory Urge for Tics Scale (PUTS): initial psychometric results and examination of the premonitory urge phenomenon in youths with Tic disorders. J Dev Behav Pediatr. 2005; 26:397-403. [PubMed: 16344654]

12. Peterson BS, Leckman JF. The temporal dynamics of tics in Gilles de la Tourette syndrome. Biol Psychiatry. 1998; 44:1337-48. [PubMed: 9861477]

13. Conelea CA, Woods DW. The influence of contextual factors on tic expression in Tourette's syndrome: a review. J Psychosom Res. 2008; 65:487-96. [PubMed: 18940379]

14. Himle MB, Woods DW, Conelea CA, Bauer CC, Rice KA. Investigating the effects of tic suppression on premonitory urge ratings in children and adolescents with Tourette's syndrome. Behav Res Ther. 2007; 45:2964-76. [PubMed: 17854764]

15. Gilles de la Tourette G. E'tude sur une affection nerveuse caracte'rise'e par de I'incoordination motrice accompagne'e d'echolalie et de copralalie. Archive Neurologie. 1885; 9:19-42. 158-200.

16. Tiffen, J. Purdue Pegboard Test. Chicago: Scientific Research Associates; 1968.

17. Bloch MH, Peterson BS, Scahill L, Otka J, Katsovich L, Zhang H, Leckman JF. Adulthood outcome of tic and obsessive-compulsive symptom severity in children with Tourette syndrome. Arch Pediatr Adolesc Med. 2006; 160:65-9. [PubMed: 16389213] 
18. Spencer T, Biederman M, Coffey B, Geller D, Wilens T, Faraone S. The 4-year course of tic disorders in boys with attention-deficit/ hyperactivity disorder. Arch Gen Psychiatry. 1999; 56:842-7. [PubMed: 12884890]

19. Bloch MH, Sukhodolsky DG, Leckman JF, Schultz RT. Fine-motor skill deficits in childhood predict adulthood tic severity and global psychosocial functioning in Tourette's syndrome. J Child Psychol Psychiatry. 2006; 47:551-9. [PubMed: 16712631]

20. Bloch MH, Leckman JF, Zhu H, Peterson BS. Caudate volumes in childhood predict symptom severity in adults with Tourette syndrome. Neurology. 2005; 65:1253-8. [PubMed: 16247053]

21. Singer HS. Discussing outcome in Tourette syndrome. Arch Pediatr Adolesc Med. 2006; 160:1035. [PubMed: 16389220]

22. Kalanithi PS, Zheng W, Kataoka Y, DiFiglia M, Grantz H, Saper CB, et al. Altered parvalbuminpositive neuron distribution in basal ganglia of individuals with Tourette syndrome. Proc Natl Acad Sci U S A. 2005; 102:13307-12. [PubMed: 16131542]

23. Leckman JF, Vaccarino FM, Kalanithi PS, Rothenberger A. Annotation: Tourette syndrome: a relentless drumbeat—driven by misguided brain oscillations. J Child Psychol Psychiatry. 2006; 47:537-50. [PubMed: 16712630]

24. Gerard E, Peterson BS. Developmental processes and brain imaging studies in Tourette syndrome. J Psychosom Res. 2003; 55:13-22. [PubMed: 12842227]

25. Albin RL. Neurobiology of basal ganglia and Tourette syndrome: striatal and dopamine function. Adv Neurol. 2006; 99:99-106. [PubMed: 16536355]

26. Scahill L, Erenberg G, Berlin CM, Budman C, Coffey BJ, Jankovic J, et al. Contemporary assessment and pharmacotherapy of Tourette syndrome. NeuroRx. 2006; 3:192-206. [PubMed: 16554257]

27. Bloch MH. Emerging treatments for Tourette's disorder. Curr Psychiatry Rep. 2008; 10:323-30. [PubMed: 18627671]

28. Walkup, JT.; Khan, S.; Schuerholz, L.; Paik, Y-S.; Leckman, JF.; Schultz, RT. Phenomenology and natural history of tic-related ADHD and learning disabilities. In: Leckman, JF.; Cohen, DJ., editors. Tourette's Syndrome Tics, Obsessions, Compulsions — Developmental Psychopathology and Clinical Care. New York: John Wiley and Sons; 1998. p. 63-79.

29. Sukhodolsky DG, Scahill L, Zhang H, Peterson BS, King RA, Lombroso PJ, et al. Disruptive behavior in children with Tourette's syndrome: association with ADHD comorbidity, tic severity, and functional impairment. J Am Acad Child Adolesc Psychiatry. 2003; 42:98-105. [PubMed: 12500082]

30. Hoekstra PJ, Steenhuis MP, Troost PW, Korf J, Kallenberg CG, Minderaa RB. Relative contribution of attention-deficit hyperactivity disorder, obsessive-compulsive disorder, and tic severity to social and behavioral problems in tic disorders. J Dev Behav Pediatr. 2004; 25:272-9. [PubMed: 15308928]

31. Gorman DA, Thompson N, Plessem KJ, Robertson MM, Leckman JF, Peterson BS. A controlled study of psychosocial outcome and psychiatric comorbidity in children with Tourette syndrome followed up in late adolescence. Am J Psychiatry. submitted.

32. Faraone SV, Biederman J, Mick E. The age-dependent decline of attention deficit hyperactivity disorder: a meta-analysis of follow-up studies. Psychol Med. 2006; 36:159-65. [PubMed: 16420712]

33. Treatment of ADHD in children with tics: a randomized controlled trial. Neurology. 2002; 58:52736. [PubMed: 11865128]

34. Lowe TL, Cohen DJ, Detlor J, Kremenitzer MW, Shaywitz BA. Stimulant medications precipitate Tourette's syndrome. JAMA. 1982; 247:1168-9. [PubMed: 6120250]

35. Bloch MH, Panza KE, Landeros-Weisenberger A, Leckman JF. Meta-analysis: treatment of attention-deficit hyperactivity disorder in children with comorbid tic disorders. JAACAP. 2009; 48:884-93.

36. Leckman JF, Grice DE, Barr LC, de Vries AL, Martin C, Cohen DJ, et al. Tic-related vs. non-ticrelated obsessive compulsive disorder. Anxiety. 1994; 1:208-15. [PubMed: 9160576] 
37. March JS, Franklin ME, Leonard H, Garcia A, Moore P, Freeman J, et al. Tics moderate treatment outcome with sertraline but not cognitive-behavior therapy in pediatric obsessive-compulsive disorder. Biol Psychiatry. 2007; 61:344-7. [PubMed: 17241830]

38. Bloch MH, Landeros-Weisenberger A, Kelmendi B, Coric V, Bracken MB, Leckman JF. A systematic review: antipsychotic augmentation with treatment refractory obsessive-compulsive disorder. Mol Psychiatry. 2006; 11:622-32. [PubMed: 16585942]

39. Bloch MH, Craiglow BG, Landeros-Weisenberger A, Dombrowski PA, Panza KE, Peterson BS, et al. Predictors of early adult outcome in pediatric-onset obsessive-compulsive disorder. Pediatrics. in press.

40. Leckman JF. Tourette's syndrome. Lancet. 2002; 360:1577-86. [PubMed: 12443611]

41. Kurlan R, Whitmore D, Irvine C, McDermott MP, Como PG. Tourette's syndrome in a special education population: a pilot study involving a single school district. Neurology. 1994; 44:699702. [PubMed: 8164829]

42. Budman CL, Rockmore L, Stokes J, Sossin M. Clinical phenomenology of episodic rage in children with Tourette syndrome. J Psychosom Res. 2003; 55:59-65. [PubMed: 12842232]

43. Scahill L, Sukhodolsky DG, Bearss K, Findley D, Hamrin V, Carroll DH, et al. Randomized trial of parent management training in children with tic disorders and disruptive behavior. J Child Neurol. 2006; 21:650-6. [PubMed: 16970865] 


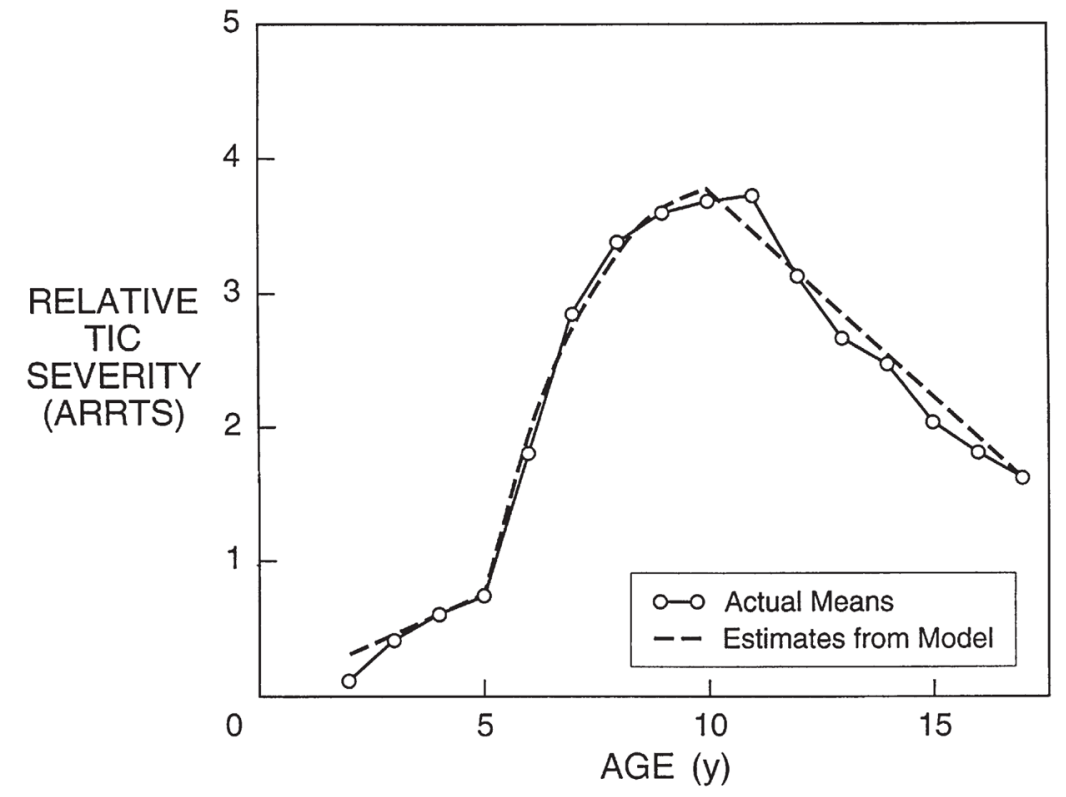

Fig. 1.

Clinical course of tic severity in childhood. Plot of average tic severity in a cohort of 36 children from ages 2 to 18 . Tics typically have an onset between ages 4 and 6 years, reach their worst-ever between ages 10 and 12, and then decline in severity throughout adolescence. (Adapted with permission from Leckman et al. [9].) ARRTS=Annual rating of relative tic severity. In the ARRTS, parents rate tic symptoms of their children on a six-point ordinal scale [absent (0), least severe, mild, moderate, severe, and most severe (6)]. 


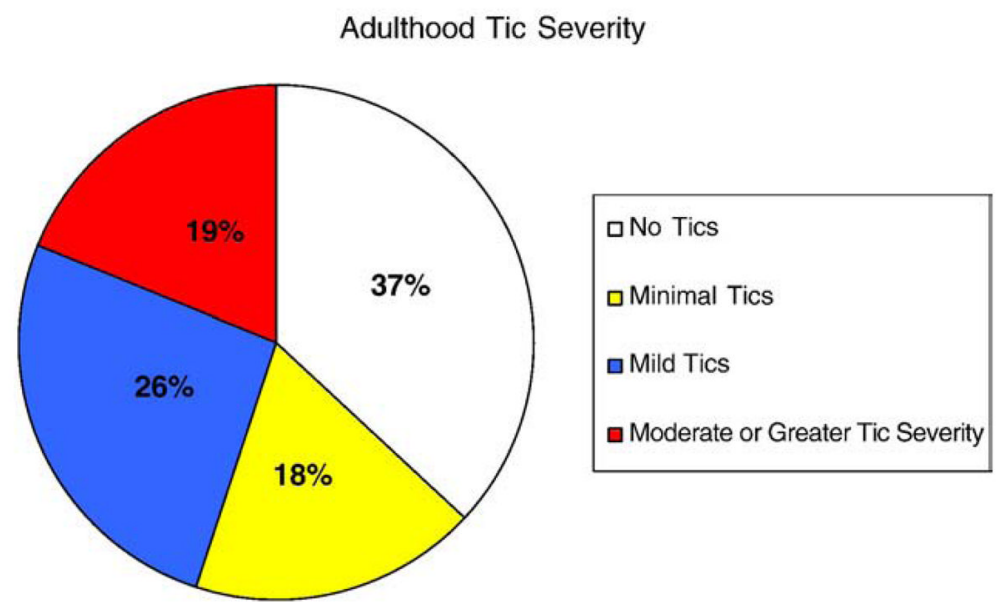

Fig. 2.

Adulthood tic severity in 82 children with significant childhood tic symptoms. Adulthood tic severity class is defined by Yale Global Tic Severity Total Tic Score (YGTSS): no tics (YGTSS: 0), minimal tics (YGTSS: 1-9), mild tics (YGTSS: 10-19), moderate or greater tics (YGTSS: 20). By contrast, all individuals had moderate or greater severity tics in childhood. Less than $5 \%$ of individuals reported having worse adulthood tics than in childhood. 


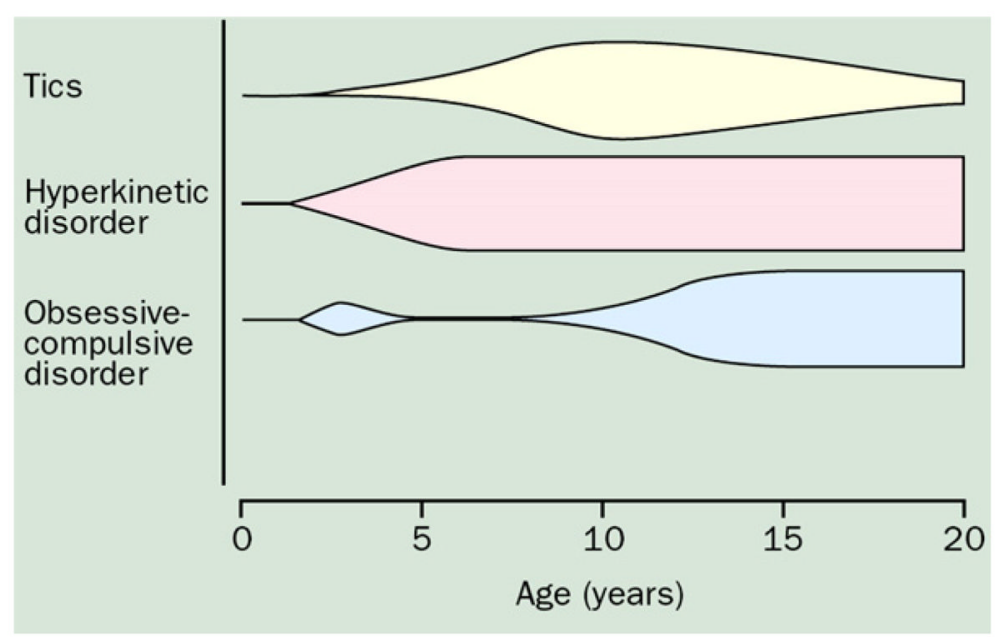

Fig. 3.

Course of Tic Severity and Comorbid Conditions in TS. The width of the bars schematically show relative burden of illnesses at a particular age. Adapted from JF Leckman 2000 [40]. 
Table 1

Psychoeducational points provided in our clinical evaluation of children with TS

Pearls of wisdom for the treatment of children with TS

1 Tics wax and wane in severity. It is important not to chase every increase in tic severity with medication and search for a medical explanation for each exacerbation.

2 Factors such as stress, fatigue, and physical illness often make tics temporarily worse. A predictable environment with appropriate sleep and hygiene is often beneficial to individuals with TS.

3 Focused concentration, especially involving motor activity, can often improve tics over the short-term. Having an activity, such as playing a musical instrument, playing sports, or dancing, is beneficial in general and can help children persevere through particularly challenging tic bouts.

4 Pharmacological treatments for tics should focus on minimizing impairment, not eliminating tics. There is no evidence that pharmacological treatments alter the long-term clinical course of TS.

5 Tics typically reach their worst-ever severity between the ages of 10 and 12 years.

6 In the vast majority of cases, tics improve during the course of adolescence.

7 Comorbid conditions are common in TS and, when present, often cause greater impairment than the tics themselves.

8 Children with TS are at high risk of developing OCD during adolescence and early adulthood. Education and aggressive behavioral treatment of OCD symptoms may be helpful in minimizing the long-term impact of OCD.

9 Substance abuse, especially with cocaine or amphetamine compounds, often worsens tics over the short-term as well as the longterm trajectory of tics.

10 The offspring of children with TS have approximately a 10\% chance of developing significant tics (assuming their partner has no family history of tics). Usually their offspring will have less severe tics than the probands as there is some regression to the mean.

11 Many adults with TS live happy, accomplished lives despite experiencing tic symptoms. 